\title{
Crianças com queixa de dificuldade escolar: avaliação das mães através da Escala Comportamental Infantil A2 de Rutter
}

\author{
Edna Maria Marturano \\ Faculdade de Medicina de Ribeirão Preto/ USP
}

\author{
Vera Lúcia Casari Parreira \\ Hospital das Clínicas de Ribeirão Preto
}

\author{
Selma Aparecida Geraldo Benzoni \\ Mestrado em Saúde Mental da FMRP/USP
}

\begin{abstract}
Tendo em vista esclarecer a associação entre problemas emocionais / comportamentais e baixo rendimento escolar, foram conduzidos dois estudos empregando a Escala Comportamental Infantil A2 de Rutter (ECI). No Estudo 1, a ECI foi aplicada a 200 mães de crianças referidas para atendimento psicológico em uma clínica-escola em razão de dificuldade no aprendizado escolar, verificando-se as diferenças entre sexos. No Estudo 2, foram avaliadas 75 crianças em três grupos: 1. com queixa de dificuldade de aprendizagem e suspeita de déficit cognitivo leve; 2 . com queixa de dificuldade de aprendizagem, sem suspeita de déficit cognitivo; 3 . com desempenho escolar satisfatório. Verificou-se uma alta porcentagem de crianças que, segundo o escore obtido na ECI, necessitariam de atendimento psicológico; as mães indicaram maior intensidade de problemas internalizados nas meninas, bem como de comportamentos imaturos nas crianças com suspeita de déficit cognitivo. A ECI confirmou tendências detectadas em estudos anteriores baseados em entrevistas.
\end{abstract}

Palavras-chave: baixo desempenho escolar; problemas de comportamento; Escala Comportamental Infantil A2

\begin{abstract}
Children with a school learning disabilities: assessment by mothers using the Rutter Child Behavior Scale-A2

With the aim of clarifying the association between behavioral / emotional problems and school underachievement, two studies were conducted with the Rutter Child Behavior Scale - A2 (CBS) In Study 1, CBS was applied to 200 mothers, each one with a child who had been referred to a university child guidance clinic for psychological aid by virtue of school learning problems; sex differences were investigated. In Study 2, seventy-four children in three groups were assessed through CBS: 1. Children with school learning problems and presumed educable mental retardation; 2. Children with school learning problems and presumed normal intelligence; 3 . Children with good school performance. Results showed a high proportion of children who, according to CBS score, were in need of psychological help. Mothers reported more internalized problems in girls and more immature behaviors in children presumed to be mentally retarded. Trends detected by earlier interview researches were confirmed by CBS.
\end{abstract}

Key words: school underachievement; behavior problems; Child Behavior Scale - A2.

Endereço para correspondência: Edna Maria Marturano: FMRP/USP - Curso de Pós-graduação em Saúde Mental, Av. 9 de Julho, 980, CEP, 14025-000, Ribeirão Preto, SP.

Apoio financeiro: FAPESP; CNPq. 
No atendimento psicológico à população infantil, a co-ocorrência de problemas de comportamento e baixo desempenho na escola pode ser um dos fatores responsáveis pela demanda motivada por dificuldades escolares. Essa associação, documentada na literatura (Hinshaw, 1992), vem sendo confirmada em. estudos realizados com crianças brasileiras (Santos, 1990; Graminha, 1992). Levantamentos baseados nas queixas das mães indicam, em crianças atendidas em serviços de saúde, com queixa de dificuldade na aprendizagem escolar, sinais de enfrentamento inadequado de situações quotidianas, com desorganização da conduta nas relações interpessoais e frente à frustração (Marturano, Linhares e Parreira, 1994).

Esses resultados sugerem que a população de crianças referidas para atendimento em serviços de saúde e clínicas-escola por motivo de dificuldade na aprendizagem escolar constitui um grupo de risco para distúrbios emocionais e de comportamento, com probabilidade de desadaptação psicossocial ao longo do desenvolvimento. Dentro de certos limites, esse risco pode ser dimensionado a partir de informações sobre a trajetória de crianças referidas por dificuldades de aprendizagem. Em um estudo de seguimento seis anos após o atendimento psicopedagógico, Degani e Marturano (1994) verificaram que $21 \%$ dos ex-clientes, agora com idades entre 14 e 17 anos, apresentavam problemas sérios como envolvimento com drogas, incidentes criminais, conflitos intensos nos relacionamentos e tentativas de suicídio.

Mediante essa constatação, é desejável que o psicólogo disponha de recursos para discriminar, na clientela constituída de crianças com dificuldades escolares, aquelas que apresentam indício de problema emocional / comportamental. A esse respeito, uma limitação das pesquisas baseadas em levantamentos de queixas é que elas não fornecem parâmetros precisos para tal identificação.

Estudos empregando a Escala Comportamental Infantil A2 para pais (ECI), traduzida e adaptada por Graminha (1994a), têm fornecido informações úteis para dimensionar os problemas de comportamento associados à queixa escolar. Graminha e Coelho (1994) verificaram que a ECI discrimina o grupo de crianças que, na avaliação dos pais, necessitam de atendimento psicológico, e estabeleceram um escore total acima de 16 como o índice que melhor caracteriza as crianças que necessitam de ajuda profissional segundo a percepção dos pais. Parreira (1995), empregando a ECI, estudou uma amostra de 30 crianças referidas para atendimento psicológico em virtude de dificuldade na aprendizagem escolar e verificou que $77 \%$ delas atingem o critério de Graminha e Coelho (1994), quanto à necessidade de atendimento psicológico. Comparando os resultados da amostra clínica com aqueles obtidos em amostra representativa da população geral na mesma faixa etária (Graminha, 1994b), Parreira observou que, apesar da maior incidência de problemas na amostra clínica, o perfil de ocorrência dos comportamentos inadequados foi semelhante nas duas amostras. Em uma pesquisa baseada em comparação entre grupos com e sem problema escolar (Marturano, Loureiro, Linhares e Machado, 1997), as crianças do grupo clínico com problema escolar foram avaliadas por suas mães na ECI como tendo mais característi- 
cas de impulsividade e humor depressivo que o grupo de escolares com bom desempenho.

Embora as investigações utilizando a ECI confirmem a presença de problemas de comportamento em crianças referidas para atendimento psicológico em virtude de dificuldade no aprendizado escolar, diversas questões permanecem em aberto. Uma delas diz respeito às diferenças entre sexos nessa população, constituída predominantemente por meninos. Outra se refere às diferenças entre subgrupos clínicos, em particular aquelas associadas ao nível cognitivo, cuja heterogeneidade nessa população foi apontada em pelo menos dois estudos (Marturano, Magna e Murtha, 1992; Loureiro e cols, 1994).

Tendo em vista esclarecer essas questões, assim como verificar, em uma amostra numericamente mais representativa, os resultados obtidos por Parreira (1995), a investigação relatada neste texto teve por objetivo identificar, através da ECI, problemas emocionais / comportamentais na clientela de um serviço especializado em problemas de aprendizagem, por meio de dois estudos, os quais são relatados nos parágrafos subseqüentes.

\section{Estudo 1}

O Estudo 1 teve o objetivo de caracterizar os problemas de comportamento presentes em uma amostra clínica referida por dificuldade na aprendizagem escolar, bem como detectar diferenças entre sexos, comparando essas informações com as encontradas por Graminha (1994b) em amostra representativa da população.

\section{Método}

\section{Sujeitos}

Foram sujeitos 200 crianças cujas famílias compareceram a uma clínica-escola através de encaminhamento ou por procura espontânea, apresentando como queixa principal o baixo rendimento escolar. As crianças tinham de sete a 11 anos, freqüentavam a primeira, segunda ou terceira série e residiam no município de Ribeirão Preto. Havia na amostra 141 meninos e 59 meninas.

\section{Instrumento}

Foi utilizada a Escala Comportamental Infantil A2 de Rutter, traduzida e adaptada por Graminha (1994a). A Escala, em sua forma adaptada, está composta por 36 itens que investigam problemas, agrupados em três sub-escalas: Saúde (oito itens), Hábitos (sete itens) e Comportamentos (21 itens). Cada item consiste numa afirmação breve, com três alternativas de resposta, conforme ilustrado nos exemplos abaixo

$\begin{array}{lccc}\begin{array}{l}\text { Sub-escala Saúde: } \\ \text { Tem queixa de dores de cabeça }\end{array} & \text { Nunca } & \begin{array}{l}\text { Ocasionalmente, não chega a } \\ \text { ocorrer uma vez por semana }\end{array} & \text { Pelo menos uma vez por semana } \\ \begin{array}{l}\text { Sub-escala Hábitos: } \\ \text { Há qualquer difículdade de alimentação? }\end{array} & 0 & 0 & \text { Sim - severa } \\ \begin{array}{l}\text { Sub-escala Comportamento: } \\ \begin{array}{l}\text { Muito agitado, tem dificuldade em per- } \\ \text { manecer sentado por muito tempo }\end{array}\end{array} & \text { Não se aplica } & \text { Se aplica um pouco } & \text { Se aplica com certeza }\end{array}$


Os itens são pontuados de acordo com os seguintes critérios: às respostas que se referem à negativa de um problema é atribuído o valor 0 (zero); às respostas que se referem à ocorrência ocasional ou moderada do problema é atribuído 0 valor 1 (um); às respostas que se referem ao aparecimento freqüente ou intenso do problema é atribuído o valor 2 (dois). Assim, a pontuação máxima que uma criança pode receber na escala é $36 \times 2=72$.

\section{Procedimento}

A coleta de dados foi inserida na rotina da clínica-escola. A ECI era apresentada individualmente à mãe durante a primeira entrevista, que tinha por finalidade o esclarecimento da queixa. Todas as entrevistas foram conduzidas por psicólogas.

\section{Resultados e discussão}

A análise dos escores totais na escala indicou que $74 \%$ das crianças têm escore total acima de 16, critério estabelecido por Graminha e Coelho (1994) para identificar crianças que, na avaliação dos pais, necessitam de atendimento psicológico. Essa porcentagem é semelhante à encontrada por Parreira (1995) em uma amostra menor de clientes do mesmo serviço.

Analisadas as sub-escalas Saúde, Hábitos e Comportamento, verificou-se que os problemas de saúde mais freqüentes foram mau humor (citado por $81 \%$ das mães) e dor de cabeça $(46 \%)$; os problemas mais freqüentes caracterizados como hábitos foram medo (44\%) dificuldades de sono (40\%), dificuldades de alimentação $(40 \%)$ e dificuldades de fala $(38 \%)$. As incidências mais altas de problemas concentraram-se na sub-escala Comportamento: em porcentagem igual ou superior a $70 \%$, as crianças foram caracterizadas como agarradas à mãe, desobedientes, irritáveis, impacientes. Em porcentagem entre 50 e $70 \%$, foram descritas como agitadas, inseguras, tímidas, dispersas, preocupadas, briguentas e destruidoras de objetos.

Para comparação item por item entre os resultados da amostra clínica e da amostra escolar (Graminha, 1994b) foram utilizados os dados da faixa de oito a nove anos, que inclui 108 sujeitos na amostra clínica e 485 na amostra de escolares. Os dados da amostra de escolares foram extraídos do artigo original de Graminha (1994b). Os resultados estão relacionados na Tabela 1, ordenados pela diferença entre as duas amostras nas incidências observadas.

Os dados da Tabela 1 indicam que a ordem de ocorrência dos itens é semelhante nas duas amostras, com maior incidência na amostra clínica em 32 dos 36 itens da ECI. Apesar dessa semelhança, verifica-se que em oito itens a diferença entre as duas amostras na incidência do problema está acima de 25 pontos percentuais. Esses resultados sugerem, na amostra clínica, uma generalização de manifestações típicas da idade, bem como discrepância em alguns comportamentos, em relação à população escolar.

A generalização de problemas na amostra clínica pode estar refletindo uma intensificação de características próprias da idade. A mera intensificação pode estar sinalizando imaturidade, identificada em crianças da mesma popu- 
Tabela 1. Porcentagem de crianças de oito e nove anos apresentando problemas de comportamento segundo a Escala Comportamental Infantil, na amostra representativa de escolares (Graminha, 1994a) e na amostra clínica com dificuldade de aprendizagem

\begin{tabular}{|c|c|c|c|}
\hline \multirow[b]{2}{*}{ Item da ECI } & \multicolumn{2}{|c|}{$\%$ de ocorrência } & \multirow[b]{2}{*}{ diferença } \\
\hline & $\begin{array}{c}\text { na amostra escolar } \\
(n=485)\end{array}$ & $\begin{array}{l}\text { na amostra clínica } \\
\quad(\mathrm{n}=108)\end{array}$ & \\
\hline dificuldade na fala & 8 & 42 & 34 \\
\hline inseguro & 31 & 64 & 33 \\
\hline impaciente, irrequieto & 41 & 74 & 33 \\
\hline agitado & 45 & 74 & 29 \\
\hline agarrado à mãe & 50 & 79 & 29 \\
\hline tristonho, angustiado & 23 & 51 & 28 \\
\hline fechado, solitário & 25 & 53 & 28 \\
\hline destrói coisas & 26 & 53 & 27 \\
\hline fica mal humorado & 60 & 82 & 2 \\
\hline facilmente irritável & 50 & 71 & 221 \\
\hline não permanece na atividade & 44 & 63 & 19 \\
\hline tímido, retraído & 50 & 69 & 19 \\
\hline rói unha & 25 & 43 & 18 \\
\hline difícil & 25 & 43 & 18 \\
\hline briga com outras crianças & 38 & 55 & 17 \\
\hline gagueja & 7 & 26 & 16 \\
\hline maltrata outras crianças & 16 & 30 & 14 \\
\hline xixi na cama / calça & 13 & 26 & 13 \\
\hline recusa ir à escola & 10 & 22 & 12 \\
\hline asma, crise respiratória & 13 & 24 & 11 \\
\hline medo & 46 & 57 & 11 \\
\hline fala palavrões & 28 & 38 & 10 \\
\hline furto/roubo & 8 & 17 & 9 \\
\hline receio de situação nova & 33 & 42 & 9 \\
\hline muito preocupado & 49 & 57 & 8 \\
\hline falta às aulas & 6 & 13 & 7 \\
\hline chupa dedo & 8 & 15 & 7 \\
\hline fala mentira & 40 & 46 & 6 \\
\hline faz cocô na roupa & 2 & 6 & 4 \\
\hline não querido por crianças & 24 & 28 & 4 \\
\hline movimento repetitivo, tique & 11 & 13 & 2 \\
\hline dor de cabeça & 44 & 46 & 2 \\
\hline desobediente & 70 & 70 & 0 \\
\hline Dor de estômago & 24 & 22 & -2 \\
\hline difícil alimentação & 47 & 44 & -3 \\
\hline dificuldade de sono & 47 & 40 & -7 \\
\hline
\end{tabular}

lação clínica por Loureiro e cols. (1994) através da avaliação com técnica projetiva gráfica. Uma explicação alternativa seria plausível a partir do pressuposto de que a situação escolar atual se configura como crise, constituindo fonte de stress se percebida pela criança como demanda que excede seus recursos de enfrentamento (Cohen e Wills, 1985). 
Quanto ao significado das reações discrepantes, as manifestações de insegurança e dependência podem estar associadas à baixa auto-estima, esperada em crianças com história de fracasso escolar e detectada por Loureiro e cols (1994). A maior incidência de dificuldade de fala nessa população foi verificada anteriormente por Marturano e cols. (1993), que interpretaram esse achado como indício de dificuldades mais amplas de linguagem, uma condição antecedente a dificuldades na aprendizagem da leitura. Os sinais de agitação e impaciência podem refletir características de temperamento, com probabilidade de afetarem o rendimento escolar (Martin, 1994); resultados de pesquisas sugerem que tais características são detectadas precocemente pelas mães, antecedendo o ingresso da criança na escola (Parreira, 1995; Magna, 1997).

No conjunto, os itens mais discrepantes em relação à população da mesma faixa etária são sugestivos da presença, na amostra clínica, de três características relevantes para a compreensão dos problemas escolares: dificuldades de linguagem que podem interferir no aprendizado da leitura, comportamentos conflitantes com as demandas da sala de aula e dificuldades emocionais.

Diferenças associadas ao sexo foram detectadas através do teste Qui-quadrado, com nível de significância de .05. Optou-se por aplicar o Qui-quadrado porque foi esse o teste estatístico usado por Graminha (1994b), cujos resultados são tomados como norma no presente estudo. De acordo com essa análise, os meninos não apresentam qualquer item com mais intensidade, ao passo que as meninas apresentam mais intensamente os itens chupa dedos, rói unhas, é inseguro.

As diferenças entre sexos não são diretamente comparáveis nas duas amostras, dado que a amostra escolar cobre um espectro maior de idades. Essa limitação deve ser considerada ao se examinarem as informações contidas na Tabela 2, onde se apresentam, para fins de comparação, os itens com diferenças entre sexos em cada amostra.

Observa-se que as diferenças entre sexos, embora com tendências semelhantes nas duas amostras, atenuam-se na amostra clínica, sobretudo naquelas características que aparecem com maior intensidade no grupo masculino. Considerando que a discrepância entre a amostra de escolares e a amostra clínica, no que se refere a diferenças entre sexos, poderia ser devida à assimetria na composição desta última, constituída por $70 \%$ de crianças do sexo masculino, a análise foi refeita reduzindo-se o grupo masculino a 59 sujeitos, através do pareamento, por idade, com sujeitos do sexo feminino. Nessa análise, empregou-se o teste $\mathrm{T}$ de Student. De acordo com os resultados, mantiveram-se as diferenças apresentadas na Tabela 2 e mais dois itens apareceram com maior intensidade entre as meninas: dor de cabeça e tímido / retraído.

Levando-se em conta a tendência evolutiva dos itens que apresentaram diferenças entre sexos na amostra escolar (Graminha, 1994b), verifica-se que, com exceção dos itens mal humorado/nervoso, rói unha e é inseguro, todos os demais mostraram tendência descendente na amostra de escolares, ou seja, quanto mais velhas as crianças, menor a incidência deles. 
Tabela 2. Escores médios por item da ECI, diferenças significativas obtidas na comparação entre sexos pelo teste Qui-quadrado e indicação dos itens com diferenças significativas na amostra escolar (Graminha, 1994b).

\begin{tabular}{|c|c|c|c|c|c|}
\hline \multirow[t]{2}{*}{ item } & \multicolumn{2}{|c|}{ escore médio } & \multirow[t]{2}{*}{$\mathrm{p}$} & \multicolumn{2}{|c|}{$\begin{array}{c}\text { Maior incidência no estu- } \\
\text { do de Graminha }\end{array}$} \\
\hline & masculino & femino & & meninos & meninas \\
\hline asma, crise respiratória & 0,37 & 0,20 & n.s. & $\mathrm{X}$ & \\
\hline fica mal humorado & 1,28 & 1,41 & n.s. & $\mathrm{X}$ & \\
\hline recusa escolar & 0,40 & 0,24 & n.s. & $\mathrm{X}$ & \\
\hline gagueira & 0,22 & 0,22 & n.s. & $\mathrm{X}$ & \\
\hline dificuldade de fala & 0,60 & 0,41 & n.s. & $\mathrm{X}$ & \\
\hline agitado & 1,25 & 1,00 & n.s. & $\mathrm{X}$ & \\
\hline impaciente, irrequito & 1,32 & 1,03 & n.s. & $\mathrm{X}$ & \\
\hline briga com crianças & 0,82 & 0,88 & n.s. & $\mathrm{X}$ & \\
\hline irritável & 1,37 & 1,20 & n.s. & $\mathrm{X}$ & \\
\hline chupa dedos & 0,11 & 0,39 & 0,01 & & $\mathrm{X}$ \\
\hline rói unha ou dedos & 0,48 & 0,83 & 0,01 & & $\mathrm{X}$ \\
\hline desobediente & 1,23 & 1,10 & n.s. & $\mathrm{X}$ & \\
\hline não permanece na ativ. & 1,08 & 0,86 & n.s. & $\mathrm{X}$ & \\
\hline medo de situação nova & 0,62 & 0,73 & n.s. & & $\mathrm{X}$ \\
\hline maltrata crianças & 0,35 & 0,37 & n.s. & $\mathrm{X}$ & \\
\hline fala palavrões & 0,58 & 0,49 & n.s. & $\mathrm{X}$ & \\
\hline agarrado à mãe & 1,23 & 1,27 & n.s. & & $\mathrm{X}$ \\
\hline inseguro & 1,03 & 1,30 & 0,01 & & \\
\hline
\end{tabular}

Assim, a atenuação das diferenças, na amostra clínica, pode ser devida ao fato de não haver crianças pequenas nesta amostra.

Em suma, os resultados das comparações entre sexos mostram que a maior demanda de atendimento para crianças do sexo masculino não está associada à maior intensidade de problemas de comportamento nos meninos.

\section{Estudo 2}

O Estudo 2 teve o objetivo de identificar diferenças na ECI associadas à variável nível intelectual, através da comparação de dois gru- pos clínicos com um grupo de escolares sem dificuldades na aprendizagem escolar.

\section{Método}

\section{Sujeitos}

Foram sujeitos 75 crianças de ambos os sexos, distribuídas em três grupos de 25 crianças:

Grupo 1: Crianças com suspeita de déficit cognitivo ( $\mathrm{QI}<70$ na Escala Wechsler de Inteligência para Crianças - WISC e na Escala de Maturidade Mental Columbia - EMMC), queixa de baixo rendimento escolar e atraso escolar, 
cujas famílias procuraram a mesma clínicaescola citada no Estudo 1.

Grupo 2: Crianças sem suspeita de déficit cognitivo ( $\mathrm{QI} \geq 70$ no WISC e na EMMC), com queixa de baixo rendimento e atraso escolar, cujas famílias procuraram a clínica;

Grupo 3: crianças sem suspeita de déficit cognitivo ( QI $\geq 70$ no WISC e na EMMC), com rendimento escolar satisfatório segundo indicação da professora, freqüentando série compatível com a idade cronológica.

Os grupos foram balanceados quanto a sexo e escolaridade dos pais, tomando-se por base a composição dos grupos clínicos. Em cada grupo havia 14 meninos e 11 meninas. As médias de idade eram 9 anos e 9 meses no grupo 1,9 anos e 10 meses no Grupo 2 e 9 anos e 7 meses no Grupo 3. Verificadas, através da ANO$\mathrm{VA}$, as diferenças entre os grupos nas variáveis idade e escolaridade dos pais, não houve diferenças significativas. Por causa do critério de atraso escolar para inclusão nos grupos 1 e 2, os grupos diferiram significativamente quanto à escolaridade dos sujeitos.

\section{Instrumentos e medidas}

Para a composição dos grupos segundo o critério do QI, foram utilizados o WISC e a EMMC. A adaptação brasileira da Escala Comportamental Infantil A2 de Rutter, versão para pais (Graminha, 1994 a) foi usada para identificação de problemas de comportamento.

\section{Procedimento}

Os instrumentos foram apresentados individualmente aos sujeitos, por psicólogas treinadas. As crianças dos Grupos 1 e 2 foram avaliadas na clínica e as demais em suas respec- tivas escolas. As mães integrantes dos grupos clínicos responderam aos itens da escala durante a primeira entrevista na clínica; junto às mães do Grupo 3 o preenchimento da escala foi feito nas escolas.

A avaliação dos grupos clínicos foi inserida na rotina de atendimento da clínica. Por esta razão foram obtidos outros dados não analisados neste estudo e no final foi realizada uma entrevista devolutiva com a criança e a mãe com o objetivo de concluir o processo diagnóstico.

\section{Resultados e discussão}

Empregou-se a análise de variância ANOVA para comparação entre grupos nos escores gerais, nas sub-escalas Saúde, Hábitos e Comportamento e em cada ítem da escala. Para verificar quais os grupos que diferem significativamente, foi aplicado o teste Tukey. Foi considerado o nível de significância de .05 .

A ANOVA indicou que os grupos clínicos não se diferenciam nos escores gerais e que ambos têm escores significativamente mais altos que os do grupo não clínico. Esse resultado se verificou também nas três sub-escalas da ECI.

Os resultados relativos à comparação item por item entre os grupos estão resumidos na Tabela 3. A tabela mostra ainda a tendência evolutiva de cada ítem na população escolar, de acordo com a investigação de Graminha (1994 b), com a seguinte distribuição: $47 \%$ dos itens da ECI mostram tendência descendente (D) conforme aumenta a idade; $5 \%$ apresentam tendência ascendente (A); 48\% não têm tendência definida. 
Tentando traduzir os resultados representados na Tabela 3, pode-se dizer, em primeiro lugar, que o grupo de escolares indicados pelas professoras por terem bom rendimento são vistos por suas mães com menos problemas de comportamento que aqueles referidos para atendimento psicológico. Com exceção do item fala palavrão, que aparece com maior intensidade no Grupo 3, as demais diferenças indicam maior prejuizo dos grupos clínicos.

Considerando-se a tendência evolutiva dos itens que discriminam e que não discriminam os grupos clínicos em relação ao grupo não clínico, os resultados mostram que: (a) dos 13 itens que discriminam o grupo clínico com suspeita de déficit cognitivo (Grupo 1), nove mostram tendência descendente na população escolar; (b) dos 9 itens que discriminam o grupo clínico sem suspeita de déficit cognitivo (Grupo 2), apenas dois apresentam tendência descendente. Esses resultados ajudam a esclarecer os dados obtidos no Estudo 1, que sugeriram maior incidência de problemas na amostra clínica em quase todos os itens da ECI. Eles parecem apoiar a hipótese de imaturidade nas crianças com queixa de dificuldade no aprendizado es-

Tabela 3. Resultados médios e comparativos entre os grupos, nos itens da escala onde houve diferença significativa; tendência evolutiva de cada item na população escolar (Graminha, 1994 b)

\begin{tabular}{|c|c|c|c|c|c|c|c|c|}
\hline \multirow[t]{2}{*}{ item } & \multicolumn{3}{|c|}{ médias* } & \multirow[t]{2}{*}{$\mathrm{F}$} & \multicolumn{3}{|c|}{$\mathrm{p}$} & \multirow{2}{*}{$\begin{array}{l}\text { tendência } \\
\text { evolutiva }\end{array}$} \\
\hline & G1 & $\mathrm{G} 2$ & G3 & & G1XG2 & G1XG3 & G2XG3 & \\
\hline dor de cabeça & 1,00 & 1,16 & 0,56 & 4,18 & $\mathrm{~ns}$ & $\mathrm{~ns}$ & 0,02 & $\mathrm{~A}^{* *}$ \\
\hline dor de estômago, vômito & 0,48 & 0,76 & 0,16 & 4,75 & $\mathrm{~ns}$ & $\mathrm{~ns}$ & 0,01 & - \\
\hline xixi na cama / calças & 0,48 & 0,28 & 0,08 & 2,87 & $\mathrm{~ns}$ & 0,05 & $\mathrm{~ns}$ & $D^{* *}$ \\
\hline fica mal humorado & 1,48 & 1,52 & 0,80 & 8,55 & $\mathrm{~ns}$ & 0,003 & 0,001 & - \\
\hline recusa ir à escola & 0,48 & 0,08 & 0,00 & 6,58 & 0,02 & 0,003 & $\mathrm{~ns}$ & $\mathrm{D}$ \\
\hline dificuldade na fala & 0,52 & 0,44 & 0,12 & 3,22 & $\mathrm{~ns}$ & 0,05 & $\mathrm{~ns}$ & $\mathrm{D}$ \\
\hline furto / roubo & 0,40 & 0,08 & 0,04 & 5,47 & 0,03 & 0,01 & $\mathrm{~ns}$ & $\mathrm{D}$ \\
\hline dificuldade de sono & 0,32 & 0,76 & 0,52 & 3,33 & 0,05 & $\mathrm{n} \mathrm{s}$ & $\mathrm{ns}$ & - \\
\hline medo & 1,08 & 0,76 & 0,52 & 3,88 & $\mathrm{~ns}$ & 0,02 & $\mathrm{~ns}$ & $\mathrm{D}$ \\
\hline impaciente, irrequieto & 1,36 & 1,16 & 0,72 & 4,01 & $\mathrm{~ns}$ & 0,02 & $\mathrm{~ns}$ & $\mathrm{D}$ \\
\hline facilmente irritável & 1,36 & 1,20 & 0,48 & 5,49 & $\mathrm{~ns}$ & 0,001 & 0,005 & D \\
\hline tristonho, angustiado & 0,64 & 0,80 & 0,24 & 4,12 & $\mathrm{~ns}$ & $\mathrm{~ns}$ & 0,02 & - \\
\hline desobediente & 1,28 & 0,92 & 0,36 & 9,07 & $\mathrm{n} \mathrm{s}$ & 0,0001 & 0,05 & $\mathrm{D}$ \\
\hline não permanece na atividade & 1,28 & 0,92 & 0,48 & 6,40 & $\mathrm{~ns}$ & 0,002 & $\mathrm{~ns}$ & $\mathrm{D}$ \\
\hline fala mentira & 0,84 & 0,60 & 0,08 & 8,71 & $\mathrm{n} \mathrm{s}$ & 0,0001 & 0,02 & - \\
\hline fala palavrões & 0,72 & 0,40 & 1,48 & 12,8 & $\mathrm{~ns}$ & 0,003 & 0,0001 & - \\
\hline agarrado à mãe & 1,56 & 1,56 & 0,88 & 5,41 & $\mathrm{~ns}$ & $\mathrm{~ns}$ & 0,01 & - \\
\hline timido, retraido & 0,96 & 0,96 & 0,44 & 3,52 & $\mathrm{~ns}$ & $\mathrm{~ns}$ & $\mathrm{~ns}$ & $\mathrm{D}$ \\
\hline inseguro & 1,12 & 1,12 & 0,60 & 4,23 & $\mathrm{~ns}$ & 0,03 & $\mathrm{~ns}$ & - \\
\hline
\end{tabular}

* G1 = Grupo clínico com suspeita de déficit cognitivo discreto; G2 = Grupo clínico sem suspeita de déficit cognitivo;

G3 = grupo não clínico

** $\mathrm{A}=$ incidência aumenta com a idade; $\mathrm{D}=$ incidência diminui com a idade. 
colar, indicando que essa imaturidade pode ser característica do grupo com suspeita de déficit cognitivo.

Tomando como referência o grupo de crianças sem dificuldade escolar, é possível traçar um perfil para cada um dos grupos clínicos, assim como identificar aspectos emocionais/ comportamentais em que ambos se assemelham. As crianças referidas para atendimento psicológico por dificuldades na aprendizagem escolar, sem suspeita de déficit cognitivo (Grupo 2), caracterizam-se por apresentarem, com mais intensidade, problemas psicossomáticos ou internalizados - dores de cabeça, dor de estômago / vômitos, dificuldades no sono; elas parecem mais tristonhas e angustiadas e são mais agarradas à mãe. Nenhum desses itens que discriminam o grupo clínico sem suspeita de déficit é sugestivo de imaturidade. Já as crianças com suspeita de déficit cognitivo segundo os resultados de duas escalas de inteligência (Grupo 1) diferenciam-se por darem trabalho ao chegar na escola ou se recusarem a entrar na escola, bem como por fazerem xixi na cama e apresentarem, com mais intensidade, dificuldade na fala, medo, irrequietude, dispersão e furto. Todos esses problemas estão no rol dos que mostram tendência descendente com a idade na população escolar.

Ambos os grupos se diferenciam do Grupo 3 em quatro características de comportamento, das quais duas apresentam tendência evolutiva descendente. Essas características comuns estão ligadas ao humor - mau humor, irritabilidade - e à sociabilidade - desobediencia, mentira.

Em síntese, a comparação entre grupos mostrou que os grupos clínicos não se diferenciam quanto ao escore global na ECI, porém apresentam perfis diversos de problemas. O grupo sem suspeita de déficit cognitivo exibe, a par com certa inadequação nas relações interpessoais, problemas de origem aparentemente emocional: queixas somáticas, dependência e reações sugestivas de ansiedade e depressão, não associadas à idade; o grupo com suspeita de déficit cognitivo também apresenta manifestações emocionais, porém típicas de crianças mais jovens, denotando imaturidade.

\section{Considerações finais}

Esta investigação teve por objetivo identificar, através da ECI, problemas emocionais / comportamentais na clientela de um serviço especializado em problemas de aprendizagem, visando não apenas determinar a incidência de problemas específicos nessa população, mas também verificar diferenças entre sexos e entre grupos clínicos com e sem suspeita de déficit cognitivo.

Os resultado obtidos, de modo geral, apoiam os de estudos anteriores com escolares e com amostras clínicas menores, indicando que esse instrumento é sensível para detectar problemas adaptativos coexistentes com a queixa escolar.

De acordo com os resultados obtidos no Estudo 1, uma porcentagem de crianças comparável àquela encontrada por Parreira (1995) atinge a "pontuação de corte" indicativa da necessidade de atendimento psicológico segundo a avaliação dos pais (Graminha e Coelho, 1994). O percentual de crianças preenchendo esse critério é elevado, sugerindo que a busca de ajuda psicológica pode estar sendo determinada não somente pela queixa escolar, mas pela coexistência entre dificuldades escolares e proble- 
mas adaptativos ocorrendo no ambiente familiar.

Segundo a percepção das mães, parecem caracterizar o funcionamento das crianças por ocasião da procura do serviço: mau humor e irritabilidade; dependência, retraimento e insegurança; agitação, irrequietude e dificuldade de concentração. Nesse perfil, as manifestações externalizadas se assemelham àquelas identificadas em estudos que se basearam no relato materno durante entrevistas: nervosismo / irritação e agitação (Parreira, 1995; Marturano, Linhares e Parreira, 1994). Já as características menos ostensivas de dependência, retraimento, insegurança e dificuldade de concentração não aparecem com a mesma ênfase nos estudos com entrevistas. Marturano, Linhares e Parreira (1994) assinalam que "reações ostensivas são alvo muito mais freqüente das referências das mães durante as entrevistas, o que é compreensível, pois não apenas são mais facilmente observáveis como também interferem mais com o meio, tendo maior probabilidade de serem avaliadas como problemáticas." As autoras acrescentam que "o profissional deve estar atento para a investigação daqueles aspectos menos visíveis, ligados à ansiedade, esquiva, retraimento, apatia - para os quais a família pode não atentar e que, não obstante, podem representar limitações do ponto de vista adaptativo" (p. 171). Os resultados do Estudo 1 mostraram que o formato da ECI, com a apresentação de ítens de comportamento a serem assinalados, pode ser um recurso ágil para o profissional evocar a reflexão das mães sobre esses aspectos. Por outro lado, é prudente examinar a possibilidade de o instrumento funcionar como um indutor, sugerindo respostas da mãe antes que evocando-as. A esse respeito, um estudo recente que utilizou o HTP, uma técnica projetiva, detectou em crianças dessa população clínica sentimentos de inferioridade e menos valia, relacionados a vivências depressivas, "com o consumo de muitos esforços defensivos para proteger-se da insegurança..." (Marturano e cols., 1997). Tais características de funcionamento interno parecem compatíveis com as avaliações maternas feitas através da ECI, de dependência, retraimento, insegurança e dificuldade de concentração, o que torna menos importante a hipótese de uma indução de respostas e fortalece a impressão de que os itens da escala ajudam a mãe a refletir sobre manifestações menos visíveis da criança.

Os resultados relativos a diferenças entre sexos, que não correspondem ao padrão verificado na população escolar, podem ser cotejados com achados de dois estudos recentes realizados em clínicas-escola, baseados em levantamentos de queixas. Graminha e Martins (1994) verificaram que a maior parte dos problemas de comportamento explícitos, como agressividade e irritabilidade, são alvo de queixas das mães para as crianças de ambos os sexos, ao passo que os problemas do tipo internalizado aparecem com mais freqüência no grupo de crianças do sexo feminino. Barbosa e Silvares (1994) verificaram que, isoladamente, as queixas mais freqüentes foram as mesmas para meninos e meninas, porém quando os sexos foram comparados quanto a grupos de queixas, "foi identificada uma significativa predominância masculina das queixas ligadas a distúrbios do desenvolvimento de habilidades escolares, e do sexo feminino nos distúrbios de comportamentos não-explícitos e nos distúrbios orgânicos" (p. 54). Em relação ao nosso estudo, pode-se supor que, igualados os grupos masculino e fe- 
minino quanto à queixa escolar, persistiram as diferenças relativas a problemas de comportamento internalizados e sintomas somáticos, mas freqüentes entre as meninas.

Avançando um pouco em relação aos estudos precedentes, esta investigação permitiu diferenciar dois subgrupos de importância clínica - aquele constituído de crianças com suspeita de déficit cognitivo discreto e aquele formado por crianças sem indícios de imaturidade. As crianças de ambos os grupos parecem estar sob tensão, porém reagindo a ela de forma diversa.

Para concluir, algumas considerações devem ser feitas a respeito da interpretação de resultados obtidos com a ECI. Um aspecto que requer cautela na interpretação dos resultados da Escala é que estes se referem a um momento na vida da criança; apesar dos indícios obtidos em outras investigações, de que as características de comportamento precedem o problema escolar (Parreira, 1995; Magna, 1997), alguns resultados do presente estudo são concordantes com a hipótese, levantada acima, de que podese tratar de reações transitórias a situações de tensão. Manifestações semelhantes a algumas das apresentadas pelas crianças com dificuldades escolares, como agitação, irrequietude, dificuldade de concentração, dor de estômago, medo, retraimento, tristeza e angústia, têm sido tomadas como indicadores de stress infantil (Lipp e Romano, 1987).

Um segundo aspecto a ser ressalvado é que a avaliação por meio desse instrumento é restrita, deixando de focalizar aspectos relevantes do funcionamento da criança. Para uma avaliação compreensiva, é necessária uma abordagem multidimensional. A esse propósito, o estudo de Marturano e cols. (1997) demonstrou que a combinação de técnicas de avaliação psi- cológica permite uma compreensão ampliada não só das dificuldades da criança referida com queixa escolar, mas também dos seus recursos potenciais para o enfrentamento de situações quotidianas, recursos esses que devem ser ativados e mobilizados no processo de ajuda.

\section{Referências Bibliográficas}

Barbosa, J. I. e Silvares, E. F. (1994) - Uma caracterização preliminar das clínicas-escola de Fortaleza. Estudos de Psicologia, 11, 50-56.

Boyle, M. H. e Jones, S. C. (1985) Selecting measures of emotional and behavioral disorders of childhood for use in general populations. Journal of Child Psychology and Psychiatry, 26, 137159 .

Cohen, S. e Wills, T. A. (1985) Stress, social support, and the buffering hypothesis. Psychological Bulletin, 2, 310-357

Degani, I. C. C. e Marturano, E. M. (1994) Dificuldades de aprendizagem: estudo de seguimento $2^{\circ}$ Ciclo de Estudos em Saúde Mental, Resumos. Faculdade de Medicina de Ribeirão Preto, 11.

Graminha, S. S. V. (1992) Problemas emocionais/ comportamentais e nível de escolaridade da criança. Resumos de Comunicações Científicas da XXII Reunião Anual de Psicologia da SPRP, 22.

Graminha, S. S. V. (1994 ${ }^{\mathrm{a}}$ ) A Escala Comportamental Infantil de Rutter A2: estudos de adaptação e fidedignidade. Estudos de Psicologia, 11, 34-42.

Graminha, S. S. V. (1994b) Problemas emocionais/ comportamentais em uma amostra de escolares; incidência em função do sexo e idade. Psico, 25(1), 49-74..

Graminha, S. S. V. e Coelho, W. F. (1994) Problemas emocionais / comportamentais em crianças 
que necessitam ou não de atendimento psicológico ou psiquiátrico. Comunicações Científicas da XXIV Reunião Anual de Psicologia da Sociedade Brasileira de Psicologia, 263.

Graminha, S. S. e Martins, M. A. ${ }^{\circ}$ (1994) - Procura de atendimento psicológico para crianças: características da problemática relatiada pelos pais. Psico, 25(2), 53-79.

Hinshaw, S. P. (1992) Externalizing behavior problems and academic underachievement in childhood and adolescence: causal relationships and underlying mechanisms. Psychological Bulletin, 111, 127-155.

Lipp, M. N. e Romano, A. S. P. (1987) - O stress infantil. Estudos de Psicologia, 4, 43-49

Loureiro, S. R.; Marturano, E. M.; Linhares, M. B. M.; Machado, V. L. S.; e Silva, R. A. (1994) Crianças com queixa de dficuldade escolar: avaliação psicológica através da técnica gráfica. Arquivos Brasileiros de Psicologia, 46, 161-182.

Magna, J. M. (1997) - Condições de desenvolvimento antecedentes à entrada na $1^{a}$ série, em crianças com atraso escolar. Dissertação de Mestrado, USP-FMRP, 1997.

Martin, R. P.(1994) - Child temperament and common problems in schooling: hypotheses about causal connections. Journal of School Psychology, 32, 119-134.

Marturano, E. M.; Linhares, M. B. M. e Parreira, V. L. C. Problemas emocionais e comportamentais associados a dificuldades na aprendizagem escolar. Medicina Ribeirão Preto, 26, 161-175, 1993.

Marturano, E. M., Magna, J. M. e Murtha, P. C. (1992) - Contribuição ao diagnóstico das dificuldades de aprendizagem escolar. Psicopedagogia, 11, 7-15.

Marturano, E. M.; Loureiro, S. R.; Linhares, M. B. M. e Machado, V. L. S. (1997) - A avaliação psicológica pode fornecer indicadores de problemas associados a dificuldades escolares? Estudos em
Saúde Mental - 1997. Ribeirão Preto, FMRP/ CPG em Saúde Mental, 11-48.

Parreira, V. L. C. (1995) Problemas de comportamento em crianças com dificuldades de aprendizagem escolar, segundo o relato das mães. Dissertação de Mestrado, USP.

Santos, M. A. (1990) Caracterização da clientela de uma clínica psicológica da Prefeitura de São Paulo. Arquivos Brasileiros de Psicologia, 42, 79-94. 